\title{
Determinación de la tarifa óptima para el transporte público interprovincial en la ruta Riobamba - Guamote, utilizando modelos matemáticos.
}

Determination of the optimal rate for public transport on the Riobamba Guamote route, using mathematical models

Jenny Margoth Villamarín Padilla. ${ }^{1}$, Monserrath Amparo Padilla Muñoz. ${ }^{2}$ Alexandra Patricia Guerrero Godoy. ${ }^{3} \&$ José Luis Llamuca Llamuca. ${ }^{4}$

Recibido: 10-03-2019 / Revisado: 15-03-209 /Aceptado: 04-04-2019/ Publicado: 13-05-2019

\begin{abstract}
DOI: https://doi.org/10.33262/cienciadigital.v3i2.2.462
\end{abstract}
A good public transport service within the province, represents multiple benefits for the population in general, when performing the analysis aimed at determining the optimal fare to the public transport, Riobamba - Guamote route, using mathematical models, one cannot stop side the multiple factors that condition it (fixed costs, variable costs, etc.)

The main purpose is to establish the operational costs and revenues to set a suitable rate for both the carrier and the user of the service. The information was collected from the 33 partners of the Guamote Transport Cooperative, following the methodology of the National Transit Agency, which allows to set the public transport rate, this information was obtained through registration forms, interviews, expense forms of operation and kilometers traveled, with this it was possible to determine an optimal rate of $\$ 1.24$ allowing the service provider to have an adequate profit margin.

\footnotetext{
${ }^{1}$ Escuela Superior Politécnica del Chimborazo, Facultad de Administración de Empresas, Carrera de Gestión del Transporte, Riobamba, Ecuador,j_villamarín@espoch.edu.ec

${ }^{2}$ Escuela Superior Politécnica de Chimborazo, Facultad de Administración de Empresas, Carrera de Gestión del Transporte, Riobamba, Ecuador, monserrath.padilla@espoch.edu.ec

${ }^{3}$ Escuela Superior Politécnica de Chimborazo, Facultad de Administración de Empresas, Carrera de Gestión del Transporte, Riobamba, Ecuador,

${ }^{4}$ Escuela Superior Politécnica del Chimborazo, Facultad de Administración de Empresas, Carrera de Gestión del Transporte, Riobamba, Ecuador, josé.llamuca@espoch.edu.ec
} 
The scope of establishing a public transport tariff within the province is extended to all users of the same, when establishing the tariff this must be approved by the competent authorities through the homologation of tariffs.

Keywords: Public transport, mathematical models, Operational costs.

\section{Resumen.}

Un buen servicio de transporte público interprovincial representa múltiples beneficios para la población en general, al realizar el análisis tendiente a la determinación de la tarifa óptima en el transporte público interprovincial, en la ruta Riobamba - Guamote, mediante modelos matemáticos, no se puede dejar de lado los múltiples factores que condicionan la misma (costos fijos, costos variables, etc.)

El propósito principal es el establecer los costos operacionales e ingresos para fijar una tarifa adecuada tanto para el transportista como para el usuario del servicio. La información fue recopilada de los 33 socios de la Cooperativa de Transporte Guamote, siguiendo la metodología de la Agencia Nacional de Transito, que permite fijar la tarifa del transporte público, esta información se obtuvo por medio de fichas de registro, entrevistas, formularios de gastos de operación y kilómetros recorridos, con esto se logró determinar una tarifa óptima de \$1,24 permitiendo al prestador de servicio tener un margen adecuado de utilidad.

El alcance de fijar una tarifa de transporte interprovincial se extiende a todos los usuarios del mismo, al establecer la tarifa esta deberá ser aprobada por las autoridades competentes mediante la homologación de tarifas.

Palabras claves: Transporte público, modelos matemáticos, costos operacionales.

\section{Introducción.}

E El transporte es el medio de traslado importante y fundamental para el crecimiento y desarrollo de la sociedad ya que permite el traslado de personas y de carga, a pesar de ello presenta grandes problemas por la escaza planificación, regulación, y control en la prestación del servicio.

El transporte público interprovincial debe garantizar calidad en el servicio y a la vez ser una buena alternativa de traslado al proporcionar seguridad, comodidad, confort y sobre todo la tarifa debe ser equitativa para el prestador del servicio como para el usuario, enfocados en un estudio técnico que permite determinar la tarifa en el transporte público interprovincial de la ruta Riobamba - Guamote, basados en modelos matemáticos y la información actual de la operadora como la tarifa por el servicio prestado. 
La tarifa del transporte en la ruta Riobamba - Guamote en el año 2015 se encontraba en 0,80 centavos y en el año 2016 se estableció un incremento del $25 \%$ mediante acuerdos entre la Agencia Nacional de Tránsito y las operadoras sin un estudio técnico que sustente la fijación de la misma haciendo que el costo actual no sea rentable.

Para la fijación de la tarifa se debe tomar en cuenta los costos reales de operación de las unidades, el mantenimiento de la unidad a lo largo de la vida útil, los kilómetros recorridos, el número de usuarios entre otros aspectos y de esta manera se ofertará un servicio de calidad como: comodidad, seguridad, rapidez y con una tarifa justa tanto para el transportista como para el usuario, dejando de lado la manera empírica con la que se ha venido determinado la tarifa de pago al servicio prestado.

Una tarifa óptima tanto para el ofertante como para el usuario permitirá mejorar el incumplimiento de frecuencias, constaste disputa entre oferentes del transporte con los usuarios, mala prestación de servicio, unidades en condiciones obsoletas, que no permite una movilidad eficiente y segura para los usuarios.

\section{Marco teórico}

Tipos de transporte público de pasajeros

En el Reglamento de la Ley Orgánica de Transporte Terrestre, Tránsito y Seguridad Vial en su capítulo $\mathrm{V}$ hace referencia a los tipos de transporte en su sección I de los tipos de transporte público de pasajeros dicta:

Art. 61.- El servicio de transporte terrestre público de pasajeros, puede ser de los siguientes tipos:

1. Transporte colectivo. - Destinado al traslado colectivo de personas, que pueden tener estructura exclusiva o no y puedan operar sujetos a itinerario, horario, niveles de servicio y política tarifaria.

2. Transporte masivo. - Destinado al traslado masivo de personas sobre infraestructuras exclusivas a nivel, elevada o subterránea, creada específica y únicamente para el servicio; que operen sujetos a itinerario, horario, niveles de servicio y política tarifaria.

El transporte público de pasajeros, en todos sus ámbitos, se hará en rutas definidas por un origen, un destino y puntos intermedios, resultantes de un análisis técnico y un proyecto sustentado, sujetos a una tarifa fijada. 
Vehículos permitidos según la clase y ámbito del transporte terrestre

En cuanto al capítulo VI del Reglamento de la Ley Orgánica de Transporte Terrestre, Tránsito y Seguridad Vial denominado de los vehículos permitidos según la clase y ámbito del transporte terrestre manifiesta:

Art. 63.- Los servicios de transporte terrestre de acuerdo a su clase, tipo y ámbito podrán prestarse en los siguientes vehículos, cuyas características se establecerán en la reglamentación y normas INEN vigentes:

Transporte Terrestre Público:

1.1. Transporte Intracantonal. -

a) Transporte Colectivo: Buses y minibuses. Los mismos que pueden ser convencionales, de entrada, baja o piso bajo.

b) Transporte Masivo: Tranvías, monorriel, metros, trolebuses, buses articulados y buses biarticulados.

1.2. Transporte Interprovincial. - Buses y minibuses y buses tipo costa.

1.3. Transporte Intrarregional e Interprovincial. - Buses y minibuses y buses tipo costa.

1.4. Transporte Internacional y Fronterizo. - Buses.

Servicio de transporte público

En base a la Ley Orgánica de Transporte Terrestre, Tránsito y Seguridad Vial en uno de sus artículos hace referencia a que principios debe tener el servicio de transporte público como se presenta a continuación:

Art. 3.- El Estado garantizará que la prestación del servicio de transporte público se ajuste a los principios de seguridad, eficiencia, responsabilidad, universalidad, accesibilidad, continuidad y calidad, con tarifas socialmente justas.

Ámbitos del transporte

En la Ley Orgánica de Transporte Terrestre, Tránsito y Seguridad Vial en su título III de los ámbitos del transporte manifiesta en sus artículos lo siguiente:

Art. 65.- El servicio de transporte público comprende los siguientes ámbitos de operación: intracantonal, interprovincial, intrarregional, interprovincial e internacional.

Art. 67.- El servicio de transporte público interprovincial es aquel que opera dentro de los límites provinciales. La celebración de los contratos de operación, será atribución de los Gobiernos Autónomos Descentralizados Regionales o de la Agencia Nacional, en aquellas provincias que no formaren parte de una región, con sujeción a las políticas y resoluciones 
de la Agencia Nacional de Regulación y Control del Transporte Terrestre, Tránsito y Seguridad Vial y de conformidad con lo establecido en la presente Ley y su reglamento. (Asamblea Nacional del Ecuador, 2012)

\section{Sistema tarifario}

Las empresas de transporte tienen una principal fuente de ingresos como lo son las tarifas, además involucran la afluencia actual y potencial de diferentes sistemas de transporte público e influencian la actitud hacia el servicio que se presta. (Molinero M., 1997)

- Estructura tarifaria

Se divide de acuerdo a la forma en que la tarifa se relaciona con la distancia recorrida, considerando la tarifa por secciones por que la ruta está dividida en tramos o secciones.

- Nivel tarifario

Hace referencia a cuánto dinero se cobra por la utilización del transporte y está relacionado a la Cantidad de servicio, la Calidad de servicio y el Costo de la prestación del servicio.

- Forma de cobro y pago

El sistema de cobro es un aspecto fundamental a la hora de ofertar y adquirir el servicio y se lo realiza en ocasiones antes de abordar o después de abordar el vehículo.

La forma de pago se la realiza en efectivo.

\section{Marco conceptual}

La Resolución 122 - DIR - 2014 - ANT establece la "Metodología para la fijación de tarifas de transporte terrestre intracantonal o urbano" en el artículo 2 muestra los siguientes conceptos:

- Ruta. Recorrido: legalmente autorizado a la transportación pública, considerando origen y destino. (Riobamba - Guamote)

- Frecuencia: Horario o itinerario otorgado por autoridad competente, a las operadoras de transporte, para la prestación del servicio público de pasajeros o carga.

- Tarifa: Precio qué para el transporte de pasajeros, fijan las autoridades de tránsito y transporte terrestre.

- Pasajero: Es la persona que utiliza un medio de transporte para movilizarse de un lugar a otro, sin ser conductor. (Agencia Nacional de Tránsito, 2014)

Además, conceptualizaciones de ámbito financiero de las empresas de transporte. 
- Demanda de pasajeros: La demanda de transporte puede definirse como la disposición a pagar que tienen los consumidores por hacer uso de una determinada infraestructura o servicio de transporte. Esta disposición a pagar, que refleja la valoración que hacen los usuarios de dichos servicios, se obtiene a partir de sus preferencias sobre las distintas características de los mismos en comparación con otros bienes que pueden adquirir. En el caso del transporte público, la demanda de transporte muestra el número de pasajeros deseando utilizar el servicio a diferentes precios y/o tarifas entre un sitio de origen y uno de destino. (Mendieta, 2010)

- Costos financieros: Es el que se integra por los gastos derivados de allegarse fondos de financiamiento por lo cual representa las erogaciones destinadas a cubrir en moneda nacional o extranjera, los intereses, comisiones y gastos que deriven de un título de crédito o contrato respectivo, donde se definen las condiciones específicas y los porcentajes pactados; se calculan sobre el monto del capital y deben ser cubiertos durante un cierto periodo de tiempo. Incluye las fluctuaciones cambiarias y el resultado de la posición monetaria. (SlideShare, 2015)

- Costos de Inversión: Los costos de inversión, llamados también costos preoperativos, corresponden a aquellos que se incurren en la adquisición de los activos necesarios para poner el proyecto en funcionamiento, ponerlo "en marcha" u operativo. Para decirlo de una forma sencilla son todos aquellos costos que se dan desde la concepción de la idea que da origen al proyecto hasta poco antes de la producción del primer producto o servicio. (Conexion ESAN, 2016)

- Costos administrativos: Son los que se originan en el área administrativa, o sea, los relacionados con la dirección y manejo de las operaciones generales de la empresa. Como pueden ser sueldos, teléfono, oficinas generales, etc. Esta clasificación tiene por objeto agrupar los costos por funciones, lo cual facilita cualquier análisis que se pretenda realizar de ellas. (Thompson, 2008)

- Costos fijos: Son los que permanecen constantes dentro de un periodo determinado sin importar si cambia el volumen. (Acosta, 2017)

- Costos variables: Cambia o fluctúa en relación directa a una actividad o volumen dado. Dicha actividad puede ser referida a producción o ventas. (Acosta, 2017)

- Depreciación: La depreciación es una disminución o una pérdida de valor que soporta un bien en el transcurso del tiempo y de acuerdo a su uso. Este desgaste es considerado normal y afecta directamente la vida útil del bien o activo hasta que este llega a un punto en el cual es inutilizable. (Web y Empresas, 2015)

- SPPAT: El Sistema Público para Pago de Accidentes de Tránsito sustituyó al SOAT, de acuerdo a la Reforma a la Ley Orgánica de Transporte Terrestre Tránsito y Seguridad Vial, el cual lo pagan todos los vehículos a motor, sin restricción de ninguna naturaleza, sean de propiedad pública o privada. (Agencia Nacional de Transito, 2015) 


\section{Metodología}

La investigación metodológicamente se basa en un estudio cualitativo, cuantitativo, descriptivo y de campo, aplicada a los socios de la Cooperativa de Transporte Guamote, los cuales cuentan con un total de 33 unidades para la prestación del servicio, tomando como factores de inclusión a los usuarios del servicio.

El tipo de investigación es transversal, porque se la realizó en un período de tiempo determinado, permitiendo analizará los gastos y costos que genera la prestación del servicio en dicha ruta.

En cuanto a la técnica utilizada para la obtención de la información será la visita y observación al campo en base a entrevistas, cuestionarios, formularios para los gastos operativos y de kilometraje. La visita y la observación directa se realizó a cada unidad de transporte con el objetivo de asegurar el levantamiento de los datos necesarios para la investigación como: gastos, consumo de combustible, año de fabricación, capacidad, vida útil, depreciación etc. A más de ello se realizó la entrevista a los propietarios de las unidades quienes conocen con certeza el mantenimiento de sus unidades, los contratos de operación y la distribución de rutas y frecuencias de la cooperativa.

Con la información obtenida luego de aplicar los instrumentos de investigación a los socios de la Cooperativa de Transporte GUAMOTE y las proformas de las casas comercializadoras de insumos y accesorios, se obtuvo la siguiente información:

Entrevista aplicada al presidente de la cooperativa de transporte Interprovincial Guamote

- La Cooperativa de Transporte Guamote es la encargada de transportar pasajeros en la ruta Riobamba - Guamote y viceversa.

- En su totalidad la flota cuenta una sola marca de buses HINO

- La operadora cuenta con buses del año 2008 en adelante siendo en su mayoría buses del año 2014, en la actualidad se está renovando la flota vehicular para el ofrecer un servicio de calidad.

- La tarifa se ha venido fijando de manera empírica a través de acuerdos entre transportistas y autoridades competentes, no se tomó en cuenta factores que ayudan a determinar la tarifa, tales como parque automotor, costos operacionales, parámetros operacionales de la ruta etc.

- En su mayoría el estado de la vía se encuentra en buenas condiciones, y en su totalidad la vía es asfaltada.

Formulario de registro de las unidades

- La metodología según la $\mathrm{ANT} \mathrm{N}^{\circ} 122$ nos dice que para determinar el total del kilometraje recorrido que realiza la unidad durante el día, mes, año, en donde, se multiplica el total de kilometrajes del circuito por el número de ciclos, luego por los 
días laborables al mes. Que en este caso seria los 28 días al mes y los dos días restantes son destinados para mantenimiento de las unidades.

- Toda la flota cumple con un mismo recorrido, la cual parte del Terminal Intracantonal de Riobamba hacia Guamote en intervalos de tiempo de diez minutos, y logrando un recorrido de $88 \mathrm{Km}$ de ida y vuelta.

- Las unidades cumplen con un total de $264 \mathrm{Km}$ al día, esto multiplicado por los siete días a la semana nos da 1828 semanales, y a su vez esto multiplicado por las cuatro semanas del mes nos da un total $7392 \mathrm{Km}$. Cada unidad cumple con tres ciclos durante la jornada de trabajo.

- El costo promedio de las unidades que prestan el servicio de transporte público, de los costos variables es de $\$ 0,964$ centavos por kilómetro recorrido.

- Para el estudio se tomó como base la resolución $\mathrm{N}^{\circ} 122$ de la ANT, donde se detallan los principales rubros a considerar para el mantenimiento.

- El promedio diario de galones de combustible al día es de 22 galones, cada galón de diésel se encuentra a un precio de $\$ 1,037$, y cuyo costo promedio es de $\$ 0,08669$ por kilómetro recorrido.

- Las llantas que ocupan las unidades en su mayoría son de $\mathrm{R} 22,5$, el precio por unidad se encuentra a $\$ 360.00$ dólares. La cantidad necesaria para un bus, es de un juego de 6 llantas, aproximadamente el cambio de neumáticos se lo realiza cada $6000 \mathrm{Km}$. El costo promedio de neumáticos es de $\$ 0,036$ centavos por kilómetro recorrido.

- El pago de los costos de legalización de las unidades se lo realiza anualmente, para el estudio se procedió a mensualizar estos costos. El costo promedio de legalización es de $\$ 0,0033$ centavos por kilómetro recorrido. Aplicando esta fórmula se obtuvo un rendimiento anual de $88704 \mathrm{Km}$, este dato servirá para determinar el costo por kilómetro recorrido.

- Para la elaboración del rol de pagos de los trabajadores se tomó en cuenta los sueldos establecidos por el Ministerio del Trabajo, tanto para la secretaria, chofer y controlador.

- El salario del personal tiene distintos beneficios sociales como son: el sueldo, Aportes al IESS, las horas extras y los décimos.

- El costo promedio de gastos de administración es de \$0,2568 centavos por kilómetro recorrido.

- El costo promedio de los gastos administrativos es de $\$ 0,0005$ por kilómetro recorrido.

- El costo de capital es de \$ 0,1064 centavos por kilómetro de recorrido.

- El costo promedio total de costos fijos es de 0,25 ctvs. por cada kilómetro recorrido

- El costo promedio total de los costos variables es de 0,22 ctv. Por cada Kilómetro recorrido.

- El costo total del costo de capital es de $0,10 \mathrm{ctv}$. 


\section{Resultados}

Tabla 1. Cuadro resumen de costos operacionales de la operadora Costos variables

Costo por $\mathrm{Km}$

\begin{tabular}{cc}
\hline Neumáticos & 0,036 \\
\hline Combustibles & 0,090 \\
\hline $\begin{array}{c}\text { Mantenimiento correctivo y } \\
\text { preventivo }\end{array}$ & 0,096 \\
\hline TOTAL COSTOS VARIABLES & 0,222
\end{tabular}

\begin{tabular}{cc}
\hline \multicolumn{1}{c}{ Costos fijos } & \\
\hline Legalizaciones & 0,003 \\
\hline Gastos de administración (TH) & 0,257 \\
\hline Gastos administrativos ( \\
Servicios básicos) \\
\hline TOTAL COSTOS FIJOS \\
\hline COSTO DE CAPITAL \\
\hline COSTOS TOTALES \\
\hline
\end{tabular}

Fuente: Cooperativa de transporte Guamote

La operadora en promedio transporta 72 personas ida y vuelta en toda la ruta, dentro de los cuales se encuentran niños, estudiantes, tercera edad y adultos.

Para determinar el promedio de pasajeros al mes se multiplica el total de pasajeros día por los días laborables al mes, tomando en cuenta los días que son destinados para el mantenimiento de las unidades de transporte, lo que nos da un promedio de 6048 pasajeros al mes.

Tabla 2. Promedio de pasajeros día, semanal y mensual

\begin{tabular}{cccc}
$\begin{array}{c}\text { Pasajeros promedio } \\
\text { transportados ida } y \\
\text { vuelta }\end{array}$ & Ciclos & Pasajeros día & $\begin{array}{c}\text { Promedio de pasajeros } \\
\text { al mes }\end{array}$ \\
\hline 72 & 3 & 216 & 6048
\end{tabular}

Fuente: Cooperativa de transporte Guamote 
El ingreso percibido por la operadora es de $\$ 0,58 \mathrm{ctv}$. al mes por cada $\mathrm{Km}$ recorrido, cabe destacar que para el cálculo se tomó en cuenta la tarifa promedio, dentro del cual se hace mención a los pasajeros transportados, ya sean niños, adultos, tercera edad etc.

Tabla 3. Ingresos por sectores

\begin{tabular}{ccccc}
\hline Trayecto & $\begin{array}{c}\text { Promedio de } \\
\text { pasajeros al mes }\end{array}$ & $\begin{array}{c}\text { Tarifa actual por el } \\
\text { servicio(\$) }\end{array}$ & Tarifa promedio & $\begin{array}{c}\text { Ingresos mensuales } \\
\$\end{array}$ \\
\hline $\begin{array}{c}\text { Riobamba }- \\
\text { Guamote }\end{array}$ & 2646 & 1,00 & 0,892 & 2360
\end{tabular}

Fuente: Cooperativa de transporte Guamote

\section{Diagnóstico de la situación actual}

La Cooperativa de Transporte Publico Interprovincial Guamote, tiene rutas y frecuencias establecidas y otorgadas por la ANT, también cuenta con tarifas que se ha cobrado desde el año 2015

Tabla 4. Ingresos por sectores

\section{COOPERATIVA DE TRANSPORTE GUAMOTE}

\begin{tabular}{ll}
\hline Número de unidades: & 33 \\
\hline Numero de vueltas o ciclos: & 3 \\
\hline Frecuencia: & 10 minutos \\
\hline Línea: & Riobamba- Guamote y viceversa \\
\hline Horario de trabajo: & $4: 00$ am- 19:30pm \\
\hline Costo del pasaje: & $\$ 1.00$ \\
\hline Km recorrido: & 44 \\
\hline Circuito: & Abierto \\
\hline Tiempo de recorrido & 1 hora \\
\hline Vía: & Troncal de la sierra E35 \\
\hline Días que laboran: & Lunes- Domingo \\
\hline
\end{tabular}

Fuente: Cooperativa de transporte Guamote 


\section{Tarifa actual}

La tabla muestra tarifas históricas y actuales por el servicio prestado en el transporte interprovincial.

Tabla 5. Tarifas

\begin{tabular}{lll}
\hline Tramo & Tarifa al 2015 & Tarifa actual \\
\hline Riobamba- Guamote & 0,80 centavos & 1,00 dólar
\end{tabular}

Fuente: Cooperativa de transporte Guamote

\section{Calculo de la tarifa del transporte público interprovincial entre Riobamba - Guamote}

Para determinar la tarifa óptima para el servicio ofertado por el transporte público interprovincial, entre Riobamba - Guamote se debe tomar en cuenta los costos y gastos mensuales de la operadora, entre ellos tenemos:

\section{Kilometrajes}

Tabla 6. Total kilómetros de la ruta Kilometraje por unidad

\begin{tabular}{cccccc}
\hline \multicolumn{5}{c}{ Kilometraje por unidad } \\
\hline Trayecto & $\begin{array}{c}\text { Kilómetros del } \\
\text { circuito }\end{array}$ & Ciclos & Kilómetros por & Kilómetros & Kilómetros \\
semanal & día & & \\
\hline $\begin{array}{c}\text { Riobamba } \\
\text { Guamote }\end{array}$ & 88 & 3 & 264 & 1848 & 7392 \\
\hline
\end{tabular}

Fuente: Elaboración propia

Tabla 7. Total de Costos variables

\begin{tabular}{lcc}
\hline Insumo & Costo mensual & Costo por Kilómetro \\
\hline Neumáticos & 266,11 & 0,036 \\
\hline Combustible & 662,43 & 0,090 \\
\hline Mantenimiento general & 712,77 & 0,096 \\
\hline Costo total & 1641,31 & $\mathbf{0 , 2 2 2}$ \\
\hline
\end{tabular}

Fuente: Elaboración propia 


\section{Costos fijos}

Salario conductor: Se toma en cuenta el sueldo de $\$ 593,32$, El aporte patronal de $\$ 66,16$, el décimo tercer sueldo de $\$ 49,44$, Décimo cuarto sueldo de $\$ 32,167$, Fondos de reserva de $\$ 49,42$, vacaciones $\$ 24,72$ dando un costo mensual de $\$ 815,23$ lo que equivale a un costo por $\mathrm{km}$ de $\$ 0,110$.

Legalizaciones: Los rubros a considerar son todos aquellos que se pagan al año, para el normal funcionamiento de la unidad de transporte, bajo las normas reglamentos emitidos por los entes reguladores como:

Tabla 8. Legalizaciones

\begin{tabular}{lcc}
\hline Rubro & Costo anual & Costo por Km. \\
\hline Tasa SPPAT & 196,36 & 0,002 \\
\hline Tasas ANT & 43,20 & 0,000 \\
\hline RTV & 43,92 & 0,000 \\
\hline $\begin{array}{l}\text { Impuesto al } \\
\text { rodaje }\end{array}$ & 10,00 & 0,000 \\
\hline Total & & \\
\hline $\begin{array}{l}\text { Total costo } \\
\text { mensual }\end{array}$ & $\mathbf{2 4 , 4 6}$ & \\
& & \\
\hline
\end{tabular}

Fuente: Elaboración propia

\section{Gastos administrativos}

Para cubrir el total de los gastos que tiene la operadora, los socios realizan aportaciones mensuales por cada unidad, en promedio aportan $\$ 20,00$ mensuales.

\section{Total Costos fijos}

Matemáticamente la determinación de los costos fijos por kilómetro, con los datos anteriores será igual a:

$$
\$ / \mathrm{km}=\frac{\text { Salario conductor }}{\mathrm{km} \text { recorridos al mes }}
$$




$$
\$ / \mathrm{km}=\frac{\text { Legalización }}{\mathrm{km} \text { recorridos al mes }}
$$

$$
\$ / \mathrm{km}=\frac{\text { Gastos administrativos }}{\mathrm{km} \text { recorridos al mes }}
$$

Tabla 9. Total de costos fijos

\begin{tabular}{ccc}
\hline RUBRO & COSTO MENSUAL & $\begin{array}{c}\text { COSTO POR } \\
\text { KILOMETRO }\end{array}$ \\
\hline Mano de obra & 1898,45 & 0,26 \\
\hline Legalizaciones & 24,46 & 0,00 \\
\hline Gastos administrativos & 118,00 & 0,00 \\
\hline Total & & 0,26
\end{tabular}

\section{Fuente: Elaboración propia}

\section{Costo de capital}

Para determinar el costo de capital se ha tomado en cuenta el precio de la unidad de trasporte, con un aproximado de $\$ 100,000$ dólares.

$$
\text { CKanual }=\frac{\operatorname{Va} *(\mathbf{1}+\mathbf{r})^{\mathbf{n}} * \mathbf{r}-(\mathrm{Vs} * \mathbf{r})}{(\mathbf{1}+\mathbf{r})^{\mathbf{n}}-\mathbf{1}}
$$

$C K=$ Costo de capital

Va $=$ Valor comercial del vehículo para el año de estudio

$r=$ Tasa de interés real

$n=$ Vida útil del vehículo (20 años)

$V s=$ Valor de salvamento $(10 \%$ del valor comercial $)$ 
Tasa de interés real

$$
\mathbf{r}=\frac{(\mathbf{1}+\mathbf{k})}{(\mathbf{1}+\mathbf{f})}-\mathbf{1}
$$

$k=$ Tasa promedio anual de interés activa.

$F=$ Tasa promedio anual de inflación.

Tabla 10. Costo de Capital

\begin{tabular}{ccc}
\hline DETALLE & MENSUAL & $\begin{array}{c}\text { COSTO POR } \\
\text { KILÓMETRO }\end{array}$ \\
\hline Costo del capital (CK) & $\$ 786,71$ & 0,106
\end{tabular}

Fuente: Elaboración propia

Tabla 11. Total de costos operativos

\begin{tabular}{cc}
\hline COSTOS & COSTO POR KILÓMETROS \\
\hline Costos fijos & 0,261 \\
\hline Costos variables & 0,222 \\
\hline Costos capital & 0,106 \\
\hline COSTO TOTAL & 0,589
\end{tabular}

Fuente: Elaboración propia

\section{Tarifa actual, al punto de equilibrio, y la óptima}

La siguiente tabla indica la comparación de cada una de las tarifas: actual, en equilibrio y la óptima. La tarifa ÓPTIMA permite obtener una rentabilidad adecuada según el Costo de capital. 
Tabla 12. Determinación de la tarifa

\begin{tabular}{cccc}
\hline & ACTUAL & EQUILIBRIO & ÓPTIMA \\
\hline Tarifa & 1,00 & 1,06 & 1,24 \\
\hline Tarifa promedio & 0,89 & 0,94 & 1,11 \\
\hline $\begin{array}{c}\text { Ingresos por } \\
\text { kilometro }\end{array}$ & 0,558 & 0,589 & 0,695 \\
\hline $\begin{array}{c}\text { Costo por kilometro } \\
\text { Rentabilidad }\end{array}$ & 0,589 & 0,589 & 0,589 \\
\hline & $-0,031$ & 0,000 & 0,106
\end{tabular}

Fuente: Elaboración propia

\section{Tarifa al punto de equilibrio}

La tabla indica la tarifa en el punto de equilibrio en donde el costo por el servicio prestado, no tiene perdida ni ganancia.

Tabla 13. Tarifa al punto de equilibrio

\begin{tabular}{ccc}
\hline Ruta & Tarifa actual & $\begin{array}{c}\text { Tarifa al punto de } \\
\text { equilibrio }\end{array}$ \\
\hline Riobamba - Guamote & 1,00 & 1,06
\end{tabular}

\section{Fuente: Elaboración propia}

\section{Tarifa óptima}

La tabla muestra la tarifa óptima para la ruta, en base al estudio técnico realizado evidenciando el incremento de la tarifa para el servicio prestado.

Tabla 14. Tarifa óptima por el servicio

\begin{tabular}{ccc}
\hline Ruta & Tarifa actual & Tarifa óptima \\
\hline Riobamba-Guamote & 1,00 & 1,24
\end{tabular}

Fuente: Cooperativa de transporte Guamote 


\section{Comparación de la tarifa actual y la tarifa regulada}

La tabla muestra la nueva tarifa para la ruta, evidenciando el incremento técnico de la tarifa, con este estudio se lograría tener una rentabilidad aproximada de $\$ 781,42$ por cada unidad.

Tabla 15. Cuadro comparativo de las tarifas

\begin{tabular}{lcccc}
\hline Ruta & Tarifa actual & $\begin{array}{c}\text { Tarifa } \\
\text { promedio }\end{array}$ & $\begin{array}{c}\text { Tarifa al punto de } \\
\text { equilibrio }\end{array}$ & $\begin{array}{c}\text { Tarifa } \\
\text { optima }\end{array}$ \\
\hline $\begin{array}{l}\text { Riobamba }- \\
\text { Guamote }\end{array}$ & 1,00 & 1,11 & 1,06 & 1,24 \\
\hline
\end{tabular}

Fuente: Elaboración propia

\section{Tarifa Propuesta}

Después de realizar el respectivo análisis técnicos se puede evidenciar la existencia de un incremento a la tarifa por el servicio ofertado de $\$ 1,00$ a $\$ 1,24$ lo que significa que la operadora debería trabajar con esta tarifa técnica, con esto se lograría tener una rentabilidad aproximada de $\$ 781,42$ por cada unidad.

\section{CONCLUSIONES}

- La tarifa del transporte público interprovincial en la ruta Riobamba- Guamote, hasta el cantón Guamote debe ser de \$1,24.

- Los costos por kilómetros para transporte interprovincial en la ruta son: costos fijos $\$ 0,26$ centavos, costos variables $\$ 0,22$ centavos y los costos de capital $\$ 0,10$ centavos.

- El análisis de costos realizado, puede ser utilizado como un estudio técnico base para otras operadoras de la misma modalidad, ya que simplemente varía de acuerdo a las políticas internas de cada una.

- Fijar una tarifa mediante un análisis técnico busca un equilibrio entre un precio cobrado al consumidor sin exageración alguna y un justo ingreso para los ofertantes del servicio de tal manera que puedan percibir una utilidad razonable. 


\section{Referencias bibliográficas.}

Acosta, J. (18 de 10 de 2017). Definición de costos. Obtenido de https://www.gerencie.com/definicion-de-costos.html

Agencia Nacional de Tránsito. (Octubre de 2014). Metodología para la fijación de la tarifa en el transporte terrestre intracantonal. Obtenido de https://www.ant.gob.ec/index.php/transito-7/resoluciones-2014/file/2623resolucion-no-122-dir-2014-ant-metodologia-para-la-fijacion-de-tarifas-detransporte-terrestre-intracantonal-o-urbano

Agencia Nacional de Transito. (2015). Sistema público para pago por accidentes de tránsito. Obtenido de https://www.ant.gob.ec/index.php/home-2/36servicios/servicios-en-linea/1041-sppat\#.W2DssdIzbIV

Asamblea Nacional del Ecuador. (2012). Reglamento a la ley de transporte terrestre transito y seguridad vial. Obtenido de http://www.obraspublicas.gob.ec/wpcontent/uploads/downloads/2015/03/Decreto-Ejecutivo-No.-1196-de-11-06-2012REGLAMENTO-A-LA-LEY-DE-TRANSPORTE-TERRESTRE-TRANSITO-YSEGURIDAD-VIA.pdf

Bernal, C. A. (2010). Justificación metodológica. Obtenido de https://www.soloejemplos.com/ejemplos-de-justificacion-teorica-practica-ymetodologica/

Conexion ESAN. (28 de Junio de 2016). Costos de inversión. Obtenido de https://www.esan.edu.pe/apuntes-empresariales/2016/06/costos-de-inversion-y-deoperacion-en-la-formulacion-de-un-proyecto/

Econlink. (12 de 07 de 2010). Concepto de Inversión. Obtenido de https://www.econlink.com.ar/concepto-de-inversion

Mendieta, J. (26 de 04 de 2010). La demanda de transporte. Obtenido de https://es.slideshare.net/VirtualEsumer/demanda-de-trasporte

Molinero M., S. I. (1997). Planeacion Diseño Operacional y Administracion. Mexico: Universidad Autonoma del Estado de Mexico.

SlideShare. (04 de 11 de 2015). Costo de venta y costo financiero. Obtenido de https://es.slideshare.net/3409001/costo-de-venta-y-costo-financiero

Thompson, L. (04 de 2008). Tipos de costos. Obtenido de https://www.promonegocios.net/costos/tipos-costos.html 
Transito, A. N. (07 de Octubre de 2014). Resolución No. 122-DIR-2014-ANT METODOLOGÍA PARA LA FIJACIÓN DE TARIFAS DE TRANSPORTE TERRESTRE INTRACANTONAL O URBANO. Obtenido de https://www.ant.gob.ec/index.php/transito-7/resoluciones-2014/file/2623resolucion-no-122-dir-2014-ant-metodologia-para-la-fijacion-de-tarifas-detransporte-terrestre-intracantonal-o-urbano

Web y Empresas. (25 de Octubre de 2015). Depreciación. Obtenido de https://www.webyempresas.com/depreciacion/ 


\section{PARA CITAR EL ARTÍCULO INDEXADO.}

Villamarín Padilla, J., Padilla Muñoz, M., Guerrero Godoy, A., \& Llamuca Llamuca, J. (2019). Determinación de la tarifa óptima para el transporte público interprovincial en la ruta Riobamba - Guamote, utilizando modelos matemáticos. Ciencia Digital, 3(2.2), 83101. https://doi.org/10.33262/cienciadigital.v3i2.2.462

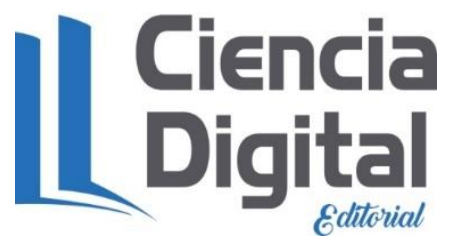

El artículo que se publica es de exclusiva responsabilidad de los autores y no necesariamente reflejan el pensamiento de la Revista Ciencia Digital.

El artículo queda en propiedad de la revista y, por tanto, su publicación parcial y/o total en otro medio tiene que ser autorizado por el director de la Revista Ciencia Digital.
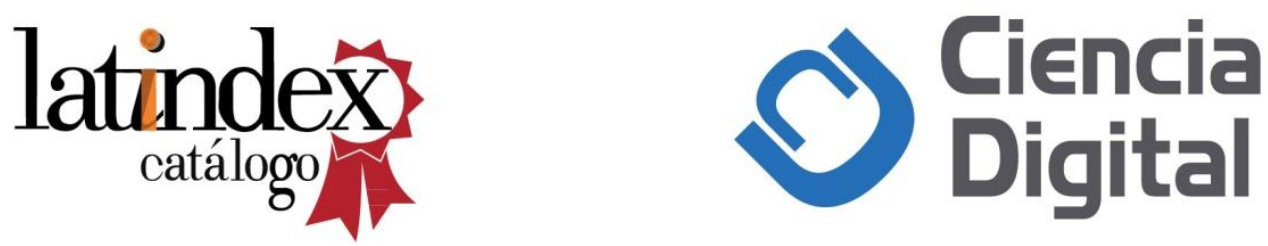